\title{
Metabolic Control of Cell Elongation and Cell Division in Bacillus subtilis
}

\author{
Anne Galinier*, Elodie Foulquier and Frédérique Pompeo \\ Laboratoire de Chimie Bactérienne, UMR 7283, CNRS/Aix-Marseille Université, Institut de Microbiologie de la Méditerranée, \\ Marseille, France
}

OPEN ACCESS

Edited by:

Martin Loose,

Institute of Science and Technology Austria (IST Austria), Austria

Reviewed by:

Dirk-Jan Scheffers,

University of Groningen, Netherlands

Imrich Barak,

Institute of Molecular Biology (SAS),

Slovakia

Petra Levin,

Washington University in St. Louis,

United States

${ }^{*}$ Correspondence:

Anne Galinier

galinier@imm.cnrs.fr

Specialty section:

This article was submitted to Microbial Physiology and Metabolism,

a section of the journal

Frontiers in Microbiology

Received: 20 April 2021

Accepted: 31 May 2021

Published: 25 June 2021

Citation:

Galinier A, Foulquier E and Pompeo F (2021) Metabolic Control of Cell Elongation and Cell Division

in Bacillus subtilis.

Front. Microbiol. 12:697930. doi: 10.3389/fmicb.2021.697930
To survive and adapt to changing nutritional conditions, bacteria must rapidly modulate cell cycle processes, such as doubling time or cell size. Recent data have revealed that cellular metabolism is a central regulator of bacterial cell cycle. Indeed, proteins that can sense precursors or metabolites or enzymes, in addition to their enzymatic activities involved in metabolism, were shown to directly control cell cycle processes in response to changes in nutrient levels. Here we focus on cell elongation and cell division in the Gram-positive rod-shaped bacterium Bacillus subtilis and we report evidences linking these two cellular processes to environmental nutritional availability and thus metabolic cellular status.

Keywords: Bacillus subtilis, metabolism, cell elongation, MreB, cell division, FtsZ

\section{INTRODUCTION}

To survive in their environment, bacteria have to rapidly adapt to nutrient availability. For example, in rich nutritional conditions, bacterial size can be increased and doubling time can be notably reduced in comparison to poor growth conditions (Schaechter et al., 1958; Sargent, 1975). This observation suggests that cell cycle processes like cell length doubling, duplication then separation of DNA, and finally cell division are highly linked to central metabolism to ensure a viable progeny (Sperber and Herman, 2017).

Bacillus subtilis is the best-characterized Gram-positive endospore-forming bacteria. This aerobic rod-shaped bacterium belongs to the Firmicute phylum. It grows by elongation along its long axis until cell length has doubled and then divides at midcell to give two identical daughter cells.

To grow, B. subtilis must synthetize the cell wall (CW) and the membrane. The main component of CW is the peptidoglycan (PG) in almost all bacteria (Egan et al., 2020). PG is a three-dimensional polymer that surrounds the surface of the cell and that is continuously remodeled during growth. It is composed of glycan chains crosslinked by short peptides and covers all the cytoplasmic or inner membrane. PG precursors like uridine diphosphate $\mathrm{N}$-acetylglucosamine (UDP-GlcNAc) and lipid II are synthesized in the cytoplasm (Mengin-Lecreulx et al., 1982; van Heijenoort, 2007). Then, lipid II, a disaccharide pentapeptide coupled to bactoprenol, is exported to be integrated into existing CW sacculus (Mengin-Lecreulx et al., 1982; van Heijenoort, 2007). More precisely, lipid II is flipped outside the cell and assembled into the existing sacculus by penicillin-binding proteins (PBPs),

Abbreviations: Fru6P, fructose 6-phosphate; Glc, glucose; GlcN6P, glucosamine-6-phosphate; GlN, glutamine; LTA, lipoteichoic acids; Man6P, D-mannose 6-phosphate; PG, peptidoglycan; TA, teichoic acid; UDP-GlcNAc, uridine diphosphate $\mathrm{N}$-acetylglucosamine; WTA, wall teichoic acids. 
enzymes that possess transglycosylases (TG) and transpeptidase (TP) activities. These enzymes are required to extend the glycan strands and construct peptide cross bridges (Errington and $\mathrm{Wu}$, 2017; Egan et al., 2020). These PBPs are well characterized and are the targets of several antibiotics (Hugonnet et al., 2016; Sharifzadeh et al., 2020). They act in collaboration with RodA and FtsW, SEDS-family TG, to enable, respectively, elongation and division of the bacterial cell (Meeske et al., 2016; Emami et al., 2017; Sjodt et al., 2020). Extracellular enzymes with autolytic activities are also necessary to permit the expansion of the PG by breaking bonds in pre-existing material. All these enzymatic activities should be regulated to allow controlled elongation of the CW during growth (Sassine et al., 2020). Being just outside the cytoplasmic membrane, PG serves as a protective layer that protects the membrane against the turgor pressure due to the high osmolarity of the cytoplasm. The PG, with the help of the cytoskeletal proteins of the MreB family that guide PG synthesis and hydrolysis, contributes to the shape of the cell (Jones et al., 2001; den Blaauwen et al., 2008). MreB proteins are related to eukaryotic actins from a structural and biochemical point of view; they reversibly polymerize depending on the binding and hydrolysis of ATP (van den Ent et al., 2001b; Esue et al., 2005). B. subtilis possesses three actin-like proteins, MreB, Mbl, and $\mathrm{MreBH}$, that possess some functional redundancies (Kawai et al., 2009). Deletions of $m r e B$ genes abolish rod-cell shape but overproduction of an MreB-like protein can compensate the absence of another (Kawai et al., 2009). Concerning the molecular role of $\mathrm{MreB}$, it was proposed that this protein polymerizes as dynamic patches and orchestrates the elongasome (DomínguezEscobar et al., 2011; Garner et al., 2011). In addition, it was recently shown that $\mathrm{MreBH}$ is essential for the activation of the major autolysin LytE and for its localization to the sites where the new PG is inserted (Carballido-López et al., 2006; Patel et al., 2020). The elongasome seems to be present only in rod-shaped bacteria, even if this point of view is debated, and directs lateral insertion of PG along the long axis of the cell to allow cylindrical growth (Szwedziak and Löwe, 2013). It is composed of a variety of enzymes (Figure 1), including MreB, MreC, MreD, RodA, RodZ, PBP2a, and PBPH (Errington, 2015; Errington and Wu, 2017; Egan et al., 2020; Patel et al., 2020).

The second major class of polymers that constitutes the bacillus CW are the teichoic acids (TAs) (Auer and Weibel, 2017; Wu et al., 2020). In many Gram-positive bacteria, there are two main forms, the wall TAs (WTA) covalently linked to the PG and lipoteichoic acids (LTA) connected to a lipid carrier. In B. subtilis, these two TAs have the same global composition and are mainly constituted of poly-[glycerol-phosphate].

Bacillus subtilis grows by cylindrical elongation and divides at the middle of the cell. This process necessitates a reorientation of CW synthesis. This process is orchestrated by a tubulin-like protein, FtsZ. This cytoskeletal protein is present in almost all bacteria. It is structurally and biochemically related to eukaryotic tubulin and its reversible polymerization is regulated by binding and hydrolysis of GTP (van den Ent et al., 2001a); it governs cell division (Aldea et al., 1990). Actually, FtsZ polymerizes to form a circumferential ring (also called Z-ring) that defines the site of cell division (or septum) (de Boer et al., 1992;
RayChaudhuri and Park, 1992). In addition, FtsZ also recruits several proteins (more than a dozen of proteins identified in B. subtilis) involved in the division machine, also called the divisome (Gamba et al., 2009; Errington and $\mathrm{Wu}$, 2017). The presence of a divisome is almost ubiquitous but its composition varies depending on bacteria; it is responsible of the constriction of inner and outer membranes and the PG synthesis at the division site (Szwedziak and Löwe, 2013). In B. subtilis, it was observed that the assembly of divisome occurs in two steps (Gamba et al., 2009). In the first step, Z-ring assembles early and concomitantly with FtsA, ZapA, and EzrA. Then, a second set of division proteins, including GpsB, FtsL, DivIB, FtsW, PBP2b, and DivIVA, are recruited to the middle of the cell. Several regulators of the divisome function have been identified; they are mostly inhibitors of FtsZ (Errington and Wu, 2017).

The aim of this mini review is to focus on how the cellular metabolism regulates cell cycle. Indeed, to spatially and temporally regulate their cell cycle according to nutritional availability, bacteria possess proteins that bind metabolites or precursors or metabolic enzymes that, in addition to their dedicated enzymatic functions, participate in cell cycle regulation. In this review, we describe metabolic proteins that regulate cell elongation (and cell shape) and cell division depending on growth conditions in the Gram-positive rodshaped bacterium B. subtilis.

\section{METABOLISM AND REGULATION OF CELL ELONGATION AND CELL SHAPE}

\section{Stimulation of PG Synthesis by GImR During Growth on Non-glycolytic Carbon Sources}

The GlmR protein (YvcK) is a good illustration of a link between carbon metabolism and cell elongation. This protein is present in many bacteria, but it has been mostly studied in B. subtilis. Although $g \operatorname{lm} R$ is dispensable during growth on glucose or glycolytic carbon sources, cells with a $\operatorname{glm} R$ deletion have abnormal shape and lyse under non-glycolytic growth conditions, like growth in the presence of Krebs cycle intermediates and substrates of pentose phosphate pathway (Görke et al., 2005). GlmR overproduction was shown to compensate the absence of MreB and its localization was similar to that of MreB suggesting a putative role in $\mathrm{CW}$ synthesis or in cell elongation (Foulquier et al., 2011). It was demonstrated that GlmR binds to UDPGlcNAc, a cytoplasmic precursor of PG synthesis (Foulquier and Galinier, 2017). However, mutations in GlmR affecting its binding to UDP-GlcNAc do not perturb bacterial growth on non-glycolytic carbon sources. In a genetic study, it was proposed that GlmR stimulates the activity of GlmS (Figure 1) and the binding of UDP-GlcNAc to GlmR could prevent this stimulation (Patel et al., 2018). GlmS is an enzyme involved in the synthesis of UDP-GlcNAc; it converts fructose 6-phosphate (Fru6P) and glutamine (GIN) into glucosamine-6-phosphate (GlcN6P) and glutamate (Badet et al., 1987). By measuring the production of GlcN6P with purified proteins, it was indeed 


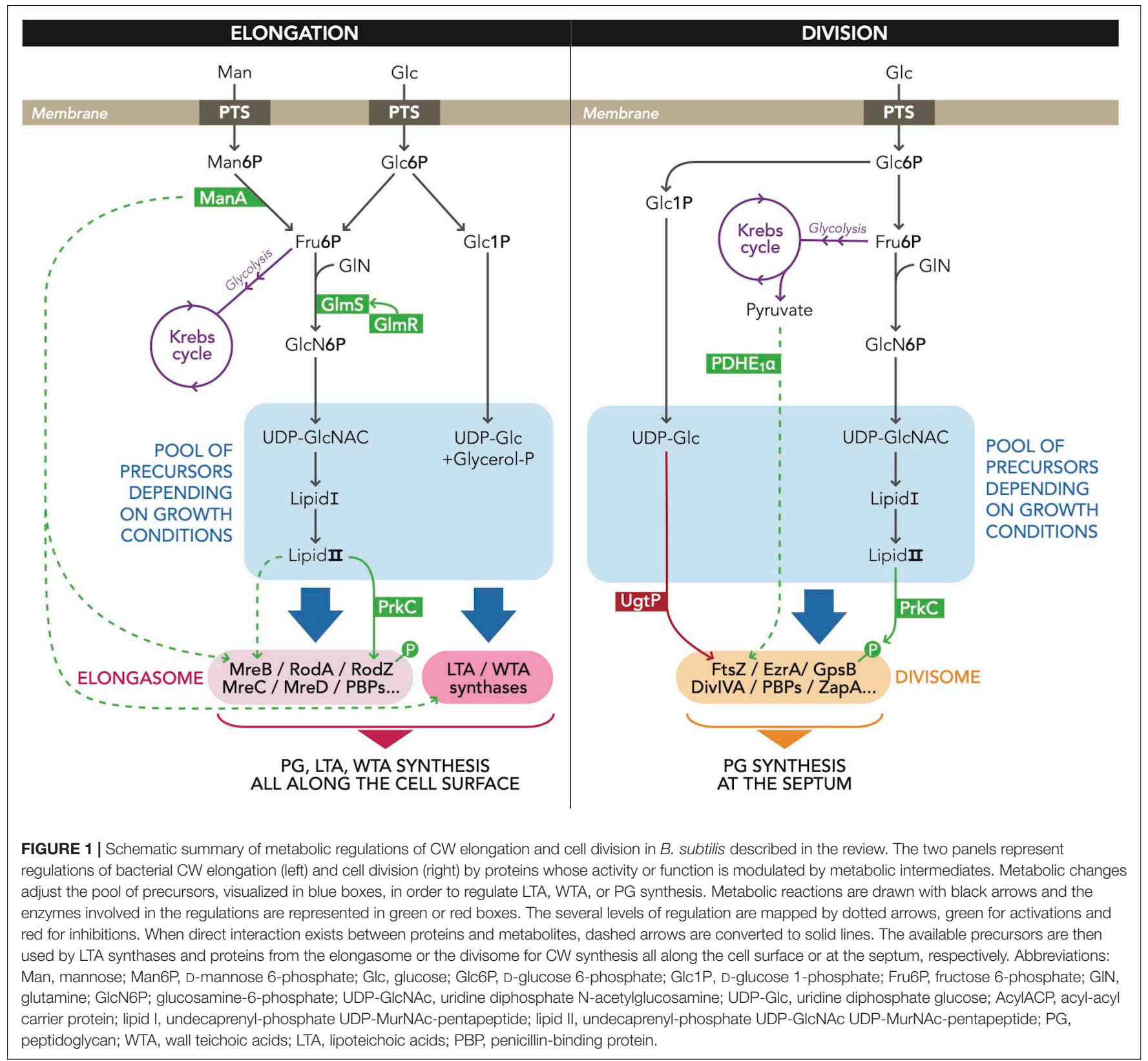

shown that GlmR directly stimulates GlmS activity and this positive effect is inhibited by UDP-GlcNAc (Foulquier et al., 2020). Combining these genetic and biochemical studies, the role of GlmR was elucidated (Foulquier and Galinier, 2017; Patel et al., 2018; Foulquier et al., 2020). When growth conditions are poor (non-glycolytic carbon sources), GlmR is essential because the intracellular concentration of UDP-GlcNAc is low. GlmR needs to interact with GlmS to stimulate GlmS activity and thus permit a normal PG synthesis and an efficient CW elongation. Indeed, during gluconeogenesis since Fru6P, the substrate of GlmS is present at low levels. The GlmR-stimulatory effect is probably essential under these conditions. In rich growth conditions, the intracellular concentration of UDP-GlcNAc is high and GlmR binds to this UDP-sugar. GlmR does not interact with GlmS and thus does not stimulate its activity. A $g \operatorname{lm} R$ deletion has thus no effect in such conditions (Patel et al., 2018; Foulquier et al., 2020).

\section{ManA: A Protein That Links Metabolism and Cell Shape}

Phosphomannose isomerase (ManA) is another protein in B. subtilis that links metabolism and cell shape. ManA is a mannose phosphate isomerase that converts D-mannose 6phosphate (Man6P) to D-Fru6P when bacteria are grown on mannose as carbon source (Sun and Altenbuchner, 2010). However, the role of this enzyme is not limited to mannose utilization. Indeed, ManA is also necessary for normal rod shape when bacteria are grown in LB medium that does not contain 
mannose. In such growth conditions, the presence of ManA avoids chromosome polyploidy and abnormal morphologies (Elbaz and Ben-Yehuda, 2010). This observation was completely unexpected and mysterious. It is noticeable that the deletion of pmi, the gene encoding a second mannose phosphate isomerase, had no observable phenotype when bacteria were grown in LB medium, indicating a specific role of ManA. The authors observed that the $\mathrm{CW}$ of the manA mutant composition has a significant decrease in the amounts of four carbohydrates that are also typical components of WTA. In contrast, the concentration of GlcNAc, the major PG carbohydrate, was similar in wild-type and $\triangle$ manA cells. Thus, the authors proposed that ManA is required for proper formation of WTA (Figure 1) and thus to maintain an equilibrium between PG and WTA; this proper balance is crucial for a correct CW formation. As a result, the deletion of manA causes an abnormal CW synthesis that induces a deregulation and an asynchrony between cell elongation, division, and nucleoid segregation (Elbaz and BenYehuda, 2010). Up to now, the molecular mechanism in which ManA is implied is uncharacterized.

\section{Regulation of MreB Dynamics by the Richness of the Growth Medium}

Other connections were observed between MreB and richness of growth conditions. Indeed, during fast growth in rich medium, bacteria need a faster synthesis of PG in comparison to slow growth in poor medium. For example, to adapt to nutrient shifts, while Escherichia coli potentially regulates the amount of PG inserted by MreB patches, B. subtilis regulates the dynamics of MreB patches (Billaudeau et al., 2017). Indeed, it was shown that the speed of MreB patches is correlated to the growth rate in $B$. subtilis and the richness of the growth medium, with a cell elongation per patch that is constant. More the medium is rich in nutrients, more the dynamics of MreB patches is high. Another recent study demonstrates that the membrane fluidity and thus MreB dynamics depend on growth conditions (Zielińska et al., 2020). This regulation is mediated by membrane proteins that form microdomains, the flotillins. These proteins may recruit specific lipids more or less rigid and whose synthesis could be dependent on the nutrient content of the growth medium. The authors observed that the addition of fluidizer is able to restore MreB dynamics and a normal cell shape to fast growing B. subtilis mutant cells that lack flotillins. They propose that, in rich medium when the bacteria are growing fast, the control of the membrane fluidity by flotillins, and thus the dynamics of MreB patches, is essential and sufficient for an efficient PG synthesis (Zielińska et al., 2020).

\section{Regulation of MreB by Lipid II}

Other studies demonstrate an additional level of regulation of MreB via the PG precursor lipid II (Figure 1) (Schirner et al., 2015). Indeed, MreB membrane association was shown to be actively regulated and to depend on the presence of cytoplasmic lipid-linked PG precursors in B. subtilis. When these PG precursors are depleted (like in poor nutrient medium), MreB patches disassemble and PG synthesis becomes anarchic. In addition, dynamics of MreB patches are still observed in mutant cells that lack WTA but synthesize PG. However, these patches alone cannot maintain a cellular rod shape. The authors proposed that the cell regulates the interaction of the MreB patches with the membrane and this level of regulation allows a rapid and reversible inactivation of $\mathrm{CW}$ enzyme complexes in response to the inhibition of CW synthesis (Schirner et al., 2015). This also suggests that the availability of precursors, and in particular lipid II, at specific sites in the cell envelope likely acts upstream of $\mathrm{MreB}$, in the spatial and temporal control of $\mathrm{CW}$ growth (Sperber and Herman, 2017). In complement to these data, a new study has very recently revealed another action of lipid II in the regulation of $\mathrm{MreB}$ via the serine/threonine kinase PrkC and the morphogenic protein RodZ (Sun and Garner, 2020). Indeed, comparing the single-cell growth rate to the density of moving MreB patches under different conditions, it was observed that the density of MreB patches is related to the growth rate. This effect requires the mur genes involved in biosynthesis of lipid II. In stationary phase, during germination and probably in poor growth conditions, the Ser/Thr kinase PrkC interacts with the lipid II or PG fragments via its extracellular domain (Shah et al., 2008; Squeglia et al., 2011; Pompeo et al., 2018). This interaction induces the oligomerization of PrkC that is evenly distributed in the CW and stimulates its kinase activity (Pompeo et al., 2018). During exponential growth in nutrientrich conditions, PrkC has a septal localization. In such conditions, its activity is not regulated via its extracellular domain and interaction with PG fragments or lipid II but it requires the division protein GpsB (Pompeo et al., 2015, 2018). PrkC has several substrates and probably connects CW state to cellular processes. In particular, it phosphorylates GlmR to regulate its activity (Foulquier et al., 2014) and also the morphogenic protein RodZ (Ravikumar et al., 2014) (Figure 1). Phosphorylated RodZ increases the density of MreB patches (Sun and Garner, 2020). As a result, the cell elongation is stimulated and the growth rate increases. In addition, an overproduction of PrkC results in cells that elongate faster than wild type in nutrient-poor conditions. The authors propose that PrkC may act as a cellular rheostat, adapting cellular processes in response to lipid II (and also probably to PG fragments), allowing cells to adjust their growth under various nutrient conditions.

\section{METABOLISM AND CELL DIVISION}

\section{Regulation of Z-Ring Formation by UDP-Glucose and UgtP}

A link between richness of growth medium and cell division was highlighted showing a direct regulation of FtsZ polymerization by a metabolic enzyme, UgtP, in B. subtilis (Weart et al., 2007). This protein acts a key metabolic regulator of cell division and is involved in glucolipids synthesis; it transfers Glc from UDPGlc to diacylglycerol-containing sugar acceptors. Deletion of the ugtP gene induces smaller cells than wild-type cells. In fact, UgtP binds to FtsZ (Figure 1) and inhibits its polymerization in a UDPGlc-dependent manner both in vivo and in vitro (Weart et al., 2007; Chien et al., 2012). In addition, it was shown that UDP-Glc, 
and consequently nutrient availability, modulates UgtP levels and localization. In summary, under rich-nutrient conditions, when UDP-Glc and UgtP intracellular concentrations are high, UgtP is localized to the septum where it binds to FtsZ to inhibit Z-ring formation. Cell division is thus delayed until the cell reaches their critical mass. Once critical mass is attained, it was proposed that a second unknown nutrient-dependent sensor alleviates this inhibition of cell division and permits progression through the remainder of the division cycle (Weart et al., 2007). In such conditions, cells are long. In poor-nutrient medium, when UDPGlc level is low, UgtP intracellular concentration is reduced and the protein is sequestered in randomly positioned foci. In such conditions, UgtP does not inhibit FtsZ polymerization and cell division occurs. Thus, the changes in UgtP levels, that depend on nutrients and are coupled with a substrate-dependent localization, link cell division with growth rate in B. subtilis (Weart et al., 2007).

\section{Regulation of Cell Division by Pyruvate and Glycolysis}

Another level of regulation of cell division by carbon metabolism was also emphasized in B. subtilis; this regulation involves glycolysis enzymes and production of pyruvate (Monahan et al., 2014). Indeed, B. subtilis strain deleted for $p y k$, the gene encoding the pyruvate kinase, an enzyme that produces pyruvate from phosphoenolpyruvate (PEP) in the final reaction of glycolysis, exhibits aberrant division events at the cell poles. The mutant cells have defects in FtsZ assembly with mislocalized Z rings. Addition of exogenous pyruvate reestablishes a normal cell division in these mutants. This observation supports the idea that pyruvate is a metabolite that plays an essential role in the coordination of bacterial cell cycle with cellular metabolic status. It was proposed that the pyruvate level is correlated to Z-ring polymerization via the $\mathrm{E} 1 \alpha$ subunit of pyruvate dehydrogenase (PDH E $1 \alpha)$, an enzyme that metabolizes pyruvate (Figure 1). Importantly, this enzyme localizes in a pyruvate-dependent manner over the nucleoid (Monahan et al., 2014). In this model, PDH E1 $\alpha$ may be involved in the stimulation of $\mathrm{Z}$ ring formation at midcell when bacteria are cultivated in nutrient-rich conditions. In such conditions, cells divide more often due to a shorter mass doubling time. The quantity of PDH E1 $\alpha$ localized within this central region increases with the augmentation of nutrient levels; this

\section{REFERENCES}

Aldea, M., Garrido, T., Pla, J., and Vicente, M. (1990). Division genes in Escherichia coli are expressed coordinately to cell septum requirements by gearbox promoters. EMBO J. 9, 3787-3794. doi: 10.1002/j.1460-2075.1990. tb07592.x

Auer, G. K., and Weibel, D. B. (2017). Bacterial cell mechanics. Biochemistry 56, 3710-3724. doi: 10.1021/acs.biochem.7b00346

Badet, B., Vermoote, P., Haumont, P. Y., Lederer, F., and LeGoffic, F. (1987). Glucosamine synthetase from Escherichia coli: purification, properties, and glutamine-utilizing site location. Biochemistry 26, 1940-1948. doi: 10.1021/ bi00381a023

Billaudeau, C., Chastanet, A., Yao, Z., Cornilleau, C., Mirouze, N., Fromion, V., et al. (2017). Contrasting mechanisms of growth in two model rod-shaped bacteria. Nat. Commun. 8:15370. doi: 10.1038/ncomms15370 could deliver a positive signal for $\mathrm{Z}$ ring formation that becomes stronger under rich conditions. However, up to now, it is not known how $\mathrm{PDH} \mathrm{E} 1 \alpha$ regulates FtsZ (directly or indirectly), how it affects cell division, and how it colocalizes with the nucleoid.

\section{CONCLUSION}

In this mini review, we focused on the well-studied Grampositive bacterium B. subtilis and showed some examples of how this bacterium adapts its cell division and elongation in response to its metabolism (Figure 1). The understanding of this adaptation is central in microbiology. Recent findings showed that metabolic enzymes play an essential role in this process. Indeed, in addition to their catalytic functions, these proteins, able to bind metabolites or precursors like UDP-Glc, UDPGlcNAc, or Lipid II, sense the metabolic status of the bacterial cell. Then they transmit this information directly to the cellcycle machineries, interacting with the elongasome, in particular with MreB, and/or with the divisome, in particular with FtsZ but not only. The discovery and the study of all those regulatory interactions that must occur to coordinate cell growth and division are a major focus in the future.

\section{AUTHOR CONTRIBUTIONS}

AG wrote the original draft. FP conceived Figure 1. AG, EF, and FP edited the manuscript. All authors contributed to the article and approved the submitted version.

\section{FUNDING}

This work was supported by the CNRS, Aix-Marseille University, and an ANR Grant (ANR-19-CE15-0011).

\section{ACKNOWLEDGMENTS}

We thank J. R. Fantino for his help in the realization of Figure 1. We also thank the reviewers for their constructive comments to improve the manuscript.

Carballido-López, R., Formstone, A., Li, Y., Ehrlich, S. D., Noirot, P., and Errington, J. (2006). Actin homolog MreBH governs cell morphogenesis by localization of the cell wall hydrolase LytE. Dev. Cell. 11, 399-409. doi: 10.1016/ j.devcel.2006.07.017

Chien, A. C., Zareh, S. K., Wang, Y. M., and Levin, P. A. (2012). Changes in the oligomerization potential of the division inhibitor UgtP co-ordinate Bacillus subtilis cell size with nutrient availability. Mol. Microbiol. 86, 594-610. doi: 10.1111/mmi.12007

de Boer, P., Crossley, R., and Rothfield, L. (1992). The essential bacterial celldivision protein FtsZ is a GTPase. Nature 359, 254-256. doi: 10.1038/359254a0

den Blaauwen, T., de Pedro, M. A., Nguyen-Distèche, M., and Ayala, J. A. (2008). Morphogenesis of rod-shaped sacculi. FEMS Microbiol. Rev. 32, 321-344. doi: 10.1111/j.1574-6976.2007.00090.x

Domínguez-Escobar, J., Chastanet, A., Crevenna, A. H., Fromion, V., WedlichSöldner, R., and Carballido-López, R. (2011). Processive movement of 
MreB-associated cell wall biosynthetic complexes in bacteria. Science 333, 225-228. doi: 10.1126/science. 1203466

Egan, A. J. F., Errington, J., and Vollmer, W. (2020). Regulation of peptidoglycan synthesis and remodelling. Nat. Rev. Microbiol. 18, 446-460.

Elbaz, M., and Ben-Yehuda, S. (2010). The metabolic enzyme ManA reveals a link between cell wall integrity and chromosome morphology. PLoS Genet 6:e1001119. doi: 10.1371/journal.pgen.1001119

Emami, K., Guyet, A., Kawai, Y., Devi, J., Wu, L. J., Allenby, N., et al. (2017). Rod a as the missing glycosyltransferase in Bacillus subtilis and antibiotic discovery for the peptidoglycan polymerase pathway. Nat. Microbiol. 2:16253. doi: $10.1038 /$ nmicrobiol.2016.253

Errington, J. (2015). Bacterial morphogenesis and the enigmatic MreB helix. Nat. Rev. Microbiol. 13, 241-248. doi: 10.1038/nrmicro3398

Errington, J., and Wu, L. J. (2017). Cell cycle machinery in Bacillus subtilis. Subcell. Biochem. 84, 67-101. doi: 10.1007/978-3-319-53047-5_3

Esue, O., Cordero, M., Wirtz, D., and Tseng, Y. (2005). The assembly of MreB, a prokaryotic homolog of actin. J. Biol. Chem. 280, 2628-2635. doi: 10.1074/jbc. M410298200

Foulquier, E., and Galinier, A. (2017). YvcK, a protein required for cell wall integrity and optimal carbon source utilization, binds uridine diphosphate-sugars. Sci. Rep. 7:4139. doi: 10.1038/s41598-017-04064-2

Foulquier, E., Pompeo, F., Bernadac, A., Espinosa, L., and Galinier, A. (2011). The YvcK protein is required for morphogenesis via localization of PBP1 under gluconeogenic growth conditions in Bacillus subtilis. Mol. Microbiol. 80, 309-318. doi: 10.1111/j.1365-2958.2011.07587.x

Foulquier, E., Pompeo, F., Byrne, D., Fierobe, H. P., and Galinier, A. (2020). Uridine diphosphate $\mathrm{N}$-acetylglucosamine orchestrates the interaction of $\mathrm{GlmR}$ with either YvcJ or GlmS in Bacillus subtilis. Sci. Rep. 10:15938. doi: 10.1038/s41598020-72854-2

Foulquier, E., Pompeo, F., Freton, C., Cordier, B., Grangeasse, C., and Galinier, A. (2014). PrkC-mediated phosphorylation of overexpressed YvcK protein regulates PBP1 protein localization in Bacillus subtilis mreB mutant cells. J. Biol. Chem. 289, 23662-23669. doi: 10.1074/jbc.M114.56 2496

Gamba, P., Veening, J. W., Saunders, N. J., Hamoen, L. W., and Daniel, R. A. (2009). Two-step assembly dynamics of the Bacillus subtilis divisome. J. Bacteriol. 191, 4186-4194. doi: 10.1128/JB.01758-08

Garner, E. C., Bernard, R., Wang, W., Zhuang, X., Rudner, D. Z., and Mitchison, T. (2011). Coupled, circumferential motions of the cell wall synthesis machinery and MreB filaments in B. subtilis. Science 333, 222-225. doi: 10.1126/science. 1203285

Görke, B., Foulquier, E., and Galinier, A. (2005). YvcK of Bacillus subtilis is required for a normal cell shape and for growth on Krebs cycle intermediates and substrates of the pentose phosphate pathway. Microbiology 151(Pt. 11), 3777-3791. doi: 10.1099/mic.0.28172-0

Hugonnet, J. E., Mengin-Lecreulx, D., Monton, A., den Blaauwen, T., Carbonnelle, E., Veckerlé, C., et al. (2016). Factors essential for L, D-transpeptidase-mediated peptidoglycan cross-linking and $\beta$-lactam resistance in Escherichia coli. Elife 5:e19469. doi: 10.7554/eLife.19469

Jones, L. J., Carballido-López, R., and Errington, J. (2001). Control of cell shape in bacteria: helical, actin-like filaments in Bacillus subtilis. Cell 104, 913-922.

Kawai, Y., Asai, K., and Errington, J. (2009). Partial functional redundancy of MreB isoforms, MreB, Mbl and MreBH, in cell morphogenesis of Bacillus subtilis. Mol. Microbiol. 73, 719-731. doi: 10.1111/j.1365-2958.2009.06805.x

Meeske, A. J., Riley, E. P., Robins, W. P., Uehara, T., Mekalanos, J. J., Kahne, D., et al. (2016). SEDS proteins are a widespread family of bacterial cell wall polymerases. Nature 537, 634-638. doi: 10.1038/nature19331

Mengin-Lecreulx, D., Flouret, B., and van Heijenoort, J. (1982). Cytoplasmic steps of peptidoglycan synthesis in Escherichia coli. J. Bacteriol. 151, 1109-1117. doi: 10.1128/jb.151.3.1109-1117.1982

Monahan, L. G., Hajduk, I. V., Blaber, S. P., Charles, I. G., and Harry, E. J. (2014). Coordinating bacterial cell division with nutrient availability: a role for glycolysis. mBio 5:e00935-14. doi: 10.1128/mBio.00935-14

Patel, V., Wu, Q., Chandrangsu, P., and Helmann, J. D. (2018). A metabolic checkpoint protein GlmR is important for diverting carbon into peptidoglycan biosynthesis in Bacillus subtilis. PLoS Genet 14:e1007689. doi: 10.1371/journal. pgen.1007689
Patel, Y., Zhao, H., and Helmann, J. D. (2020). A regulatory pathway that selectively up-regulates elongasome function in the absence of class A PBPs. Elife 9:e57902. doi: 10.7554/eLife.57902

Pompeo, F., Byrne, D., Mengin-Lecreulx, D., and Galinier, A. (2018). Dual regulation of activity and intracellular localization of the PASTA kinase PrkC during Bacillus subtilis growth. Sci. Rep. 8:1660. doi: 10.1038/s41598-01820145-2

Pompeo, F., Foulquier, E., Serrano, B., Grangeasse, C., and Galinier, A. (2015). Phosphorylation of the cell division protein GpsB regulates PrkC kinase activity through a negative feedback loop in Bacillus subtilis. Mol. Microbiol. 97, 139-150. doi: $10.1111 / \mathrm{mmi} .13015$

Ravikumar, V., Shi, L., Krug, K., Derouiche, A., Jers, C., Cousin, C., et al. (2014). Quantitative phosphoproteome analysis of Bacillus subtilis reveals novel substrates of the kinase PrkC and phosphatase PrpC. Mol. Cell. Proteomics 13, 1965-1978. doi: 10.1074/mcp.M113.035949

RayChaudhuri, D., and Park, J. T. (1992). Escherichia coli cell-division gene ftsZ encodes a novel GTP-binding protein. Nature 359, 251-254. doi: 10.1038/ $359251 \mathrm{a} 0$

Sargent, M. G. (1975). Control of cell length in Bacillus subtilis. J. Bacteriol. 123, 7-19. doi: 10.1128/jb.123.1.7-19.1975

Sassine, J., Sousa, J., Lalk, M., Daniel, R. A., and Vollmer, W. (2020). Cell morphology maintenance in Bacillus subtilis through balanced peptidoglycan synthesis and hydrolysis. Sci. Rep. 10:17910. doi: 10.1038/s41598-020-74 609-5

Schaechter, M., Maaloe, O., and Kjeldgaard, N. O. (1958). Dependency on medium and temperature of cell size and chemical composition during balanced grown of Salmonella typhimurium. J. Gen. Microbiol. 19, 592-606. doi: 10.1099/ 00221287-19-3-592

Schirner, K., Eun, Y. J., Dion, M., Luo, Y., Helmann, J. D., Garner, E. C., et al. (2015). Lipid-linked cell wall precursors regulate membrane association of bacterial actin MreB. Nat. Chem. Biol. 11, 38-45. doi: 10.1038/nchembio. 1689

Shah, I. M., Laaberki, M. H., Popham, D. L., and Dworkin, J. (2008). A eukaryotic-like Ser/Thr kinase signals bacteria to exit dormancy in response to peptidoglycan fragments. Cell 135, 486-496. doi: 10.1016/j.cell.2008. 08.039

Sharifzadeh, S., Dempwolff, F., Kearns, D. B., and Carlson, E. E. (2020). Harnessing $\beta$-lactam antibiotics for illumination of the activity of penicillinbinding proteins. ACS Chem. Biol. 15, 1242-1251. doi: 10.1021/acschembio.9b0 0977

Sjodt, M., Rohs, P. D. A., Gilman, M. S. A., Erlandson, S. C., Zheng, S., Green, A. G., et al. (2020). Structural coordination of polymerization and crosslinking by a SEDS-bPBP peptidoglycan synthase complex. Nat. Microbiol. 5, 813-820. doi: 10.1038/s41564-020-0687-z

Sperber, A. M., and Herman, J. K. (2017). Metabolism shapes the cell. J. Bacteriol. 199:e00039-17. doi: 10.1128/JB.00039-17

Squeglia, F., Marchetti, R., Ruggiero, A., Lanzetta, R., Marasco, D., Dworkin, J., et al. (2011). Chemical basis of peptidoglycan discrimination by PrkC, a key kinase involved in bacterial resuscitation from dormancy. J. Am. Chem. Soc. 133, 20676-20679. doi: 10.1021/ja208080r

Sun, T., and Altenbuchner, J. (2010). Characterization of a mannose utilization system in Bacillus subtilis. J. Bacteriol. 192, 2128-2139. doi: 10.1128/JB.016 73-09

Sun, Y., and GarnerProkaryotic origin of the actin, E. (2020). PrkC modulates MreB filament density and cellular growth rate by monitoring cell wall precursors. bioRxiv [preprint] doi: 10.1101/2020.08.28.272336

Szwedziak, P., and Löwe, J. (2013). Do the divisome and elongasome share a common evolutionary past? Curr. Opin. Microbiol. 16, 745-751. doi: 10.1016/ j.mib.2013.09.003

van den Ent, F., Amos, L. A., and Löwe, J. (2001b). Prokaryotic origin of the actin cytoskeleton. Nature 413, 39-44. doi: 10.1038/35092500

van den Ent, F., Amos, L., and Löwe, J. (2001a). Bacterial ancestry of actin and tubulin. Curr. Opin. Microbiol. 4, 634-638. doi: 10.1016/s1369-5274(01)00 $262-4$

van Heijenoort, J. (2007). Lipid intermediates in the biosynthesis of bacterial peptidoglycan. Microbiol. Mol. Biol. Rev. 71, 620-635. doi: 10.1128/MMBR. 00016-07 
Weart, R. B., Lee, A. H., Chien, A. C., Haeusser, D. P., Hill, N. S., and Levin, P. A. (2007). A metabolic sensor governing cell size in bacteria. Cell 130, 335-347. doi: $10.1016 /$ j.cell.2007.05.043

Wu, X., Han, J., Gong, G., Koffas, M. A. G., and Zha, J. (2020). Wall teichoic acids: physiology and applications. FEMS Microbiol. Rev. doi: 10.1093/femsre/fuaa064 [Epub ahead of print].

Zielińska, A., Savietto, A., de Sousa Borges, A., Martinez, D., Berbon, M., Roelofsen, J. R., et al. (2020). Flotillin-mediated membrane fluidity controls peptidoglycan synthesis and MreB movement. Elife 9:e57179. doi: 10.7554/eLife. 57179
Conflict of Interest: The authors declare that the research was conducted in the absence of any commercial or financial relationships that could be construed as a potential conflict of interest.

Copyright (๑) 2021 Galinier, Foulquier and Pompeo. This is an open-access article distributed under the terms of the Creative Commons Attribution License (CC BY). The use, distribution or reproduction in other forums is permitted, provided the original author(s) and the copyright owner(s) are credited and that the original publication in this journal is cited, in accordance with accepted academic practice. No use, distribution or reproduction is permitted which does not comply with these terms. 